BULLETIN (New Series) OF THE

AMERICAN MATHEMATICAL SOCIETY

Volume 34, Number 1, January 1997

S 0273-0979(97)00707-6

\title{
THE MATHEMATICAL ENTERPRISES OF ROBERT THOMASON
}

\author{
CHARLES A. WEIBEL
}

\begin{abstract}
During his career, Bob Thomason was involved in an interesting and varied group of mathematical endeavors. This is a retrospective survey of his contributions.
\end{abstract}

Before his death in 1995, Bob Thomason was active in many diverse areas of mathematics: homotopy theory, category theory, algebraic geometry and $K$-theory. This article will show how closely related these fields are by describing several of Thomason's more interesting mathematical endeavors. Other aspects of his career are described in his obituary [W].

\section{THE HOMOTOPY THEORY OF CATEGORIES}

Thomason's early work concerned the homotopy theory of categories. In this subject, one studies a category $C$ by analyzing the homotopy theory of its geometric realization $B C$. If Cat denotes the category of small categories, this amounts to a study of the geometric realization functor $B$ from Cat to topological spaces.

His Ph.D. dissertation [T-th] [T1] describes and analyzes homotopy colimits, using a simple but fundamental construction in category theory. Given a diagram of small categories $C_{j}$, where the subscript $j$ runs through an indexing category $J$, it is possible to construct a "canonical cofibered category" $J \int C$. Formally, this cofibered category is defined for every (lax) functor $C: J \rightarrow$ Cat. Using the geometric realization functor $B$, we can translate the data into a diagram $B C$ of topological spaces $B C_{j}$. The main result in Thomason's thesis is that the geometric realization of $J \int C$ is the homotopy colimit of the diagram $B C$. Because of the elegance and thoroughness of his analysis, this construction has become a basic tool used routinely by topologists.

One of Thomason's recurrent themes was that notions of homotopy theory were often intrinsic to Cat and also to the category Sym Cat of small symmetric monoidal categories. For example, in [T3] he proved the reassuring result that Cat admits a closed model structure, so that the abstract homotopy theory of small categories does not depend upon a need to pass to geometric realizations. In [T4] and [T5] he constructed mapping cones, mapping cylinders and other homotopy colimits within Sym Cat and showed that infinite loop space machines send these constructions to the appropriate homotopy colimits of spectra. Thomason's

Received by the editors May 30, 1996.

1991 Mathematics Subject Classification. 19-02; Secondary 18-02, 55-02.

Paper presented March 3, 1996, at The Fields Institute, Toronto.

Author partially supported by NSF grant DMS95-00791.

(C) 1997 American Mathematical Society 
constructions were central to the work of several people in the mid-1980's, including the author $[\mathrm{PW}]$.

\section{UNIQUENESS OF INFINITE LOOP SPACE MACHINES}

As he was leaving graduate school in 1977, Thomason discovered a proof that all infinite loop space machines produce equivalent output. In order to straighten out the technical details of his insight, he immediately enlisted the aid of J. Peter May. In a collaboration May recalls as "delightful interaction," they reduced Bob's argument to a characterization of infinite loop space machines by just one axiom: the "group completion" axiom. This led to the May-Thomason Uniqueness Theorem, which we shall now describe; see [MT].

For clarity, let us agree that by a spectrum $E$ we mean a sequence $\left\{E_{0}, E_{1}, \ldots\right\}$ of spaces, together with weak equivalences $E_{n} \rightarrow \Omega E_{n+1}$. Because $E_{0}$ is equivalent to the $n$-fold loop space $\Omega^{n} E_{n}$ for each $n$, we call $E_{0}$ an infinite loop space. We shall call $E$ connective if $\pi_{i} E_{n}=0$ for $i<n$.

Prior to 1977, there had been three disparate methods (called "machines") for producing infinite loop spaces and spectra: the homotopy-everything theory of Boardman and Vogt [BV], the operad-based theory of May [M] and Segal's $\Gamma$-space approach $[\mathrm{Seg}]$. These machines are characterized by their input $X$, which will be described shortly, and by their output, which should depend naturally upon the input $X$.

As output, each infinite loop space machine $E$ produces a connective spectrum $E X=\left\{E_{0} X, E_{1} X, \ldots\right\}$, together with a "group completion" map $X \rightarrow E_{0} X$.

To explain group completion, we need to know that the input $X$ has the property that $\pi_{0}(X)$ is an abelian monoid and the homology of $X$ is a graded-commutative ring. Suppose that $Y$ also has this property (e.g., that $\left.Y=E_{0} X\right)$. A map $X \rightarrow Y$ is called a (topological) "group completion" if $\pi_{0}(Y)$ is the (algebraic) group completion of the abelian monoid $\pi_{0}(X)$ and, moreover, $H_{*}(Y ; R)$ is the localization of the ring $H_{*}(X ; R)$ at the multiplicative subset $\pi_{0}(X)$ for every coefficient ring $R$.

The input is harder to describe. Although superficially very different, each machine requires that its input include a based topological space $X$, together with an action $\mathcal{G}(m, n): X^{m} \rightarrow X^{n}$ by some fixed topological family $\mathcal{G}$ of operators satisfying some simple conditions. For example, $\mathcal{G}$ should contain the projections $X^{m} \rightarrow X$ and the coordinate permutations $X^{m} \rightarrow X^{m}$, each operator $g$ in $\mathcal{G}(m, n)$ should have an underlying pointed set map $f=\epsilon g:\{0, \ldots, m\} \rightarrow\{0, \ldots, n\}$, and the subspaces $\epsilon^{-1}(f)$ of $\mathcal{G}(m, n)$ should be contractible. To verify the properties cited above about $\pi_{0}(X)$ and $H_{*}(X)$, one uses the operators $g: X^{2} \rightarrow X$.

For Segal's machine, the family of operators $\mathcal{F}$ is just the family of base-point preserving maps, so that there is a universal map $\mathcal{G} \stackrel{\epsilon}{\rightarrow} \mathcal{F}$. Segal had already observed in [Seg] that the input to any $\mathcal{G}$-based machine $E$ was equivalent to the input to his $\mathcal{F}$-based machine $S$, via the maps $\epsilon$.

The May-Thomason Uniqueness Theorem [MT]. For every infinite loop space machine $E$ there is a natural equivalence of spectra between EX and Segal's spectrum $\mathbf{B} X$.

Thomason's key idea was that one should study what happens to $E$ as $\mathcal{G}$ varies by constructing a bispectrum $\mathbf{B}_{i} E_{j} X$. This is a lattice of spaces, together with coherent equivalences $\Omega \mathbf{B}_{i+1} E_{j} X \leftarrow \mathbf{B}_{i} E_{j} X \rightarrow \Omega \mathbf{B}_{i} E_{j+1} X$. By an up-and-across 
theorem due to Fiedorowicz, the spectra $\mathbf{B}_{*} X \simeq \mathbf{B}_{*} E_{0} X$ and $E_{*} X \simeq \mathbf{B}_{0} E_{*} X$ are equivalent and the result follows.

One important application of these ideas is this. Let $S$ be a symmetric monoidal category. Its geometric realization is food for any infinite loop space machine, and the May-Thomason Uniqueness Theorem ensures that the resulting spectrum $\operatorname{Spt}(S)$ is independent of the choice of infinite loop space machine. The spectrum $\operatorname{Spt}(S)$ is sometimes called the $K$-theory spectrum of $S$, because when $S$ is the category of finitely generated projective modules over a ring $R$, the spectrum $\operatorname{Spt}(S)$ is Quillen's $K$-theory spectrum $K^{Q}(R)$. However, Thomason recently showed in [T26] that every connective spectrum is weak equivalent to $\operatorname{Spt}(S)$ for some symmetric monoidal category $S$.

\section{THOMASON'S DESCENT MACHINERY}

Following Dold's idea that homotopy theory is a kind of non-abelian homological algebra, Bousfield and Kan [BK] realized that the homotopy limit and colimit are the analogues of the derived functors of the limit and colimit.

Thomason took this philosophy one step further in 1979-80, when he developed his theory of cohomological descent for spectra [T-EC, §1]. To explain this, suppose we are given a diagram of spectra $E(j)$, i.e., a functor $j \mapsto E(j)=$ $\left\{E_{0}(j), E_{1}(j), \ldots\right\}$ from some small indexing category $J$ to the category of spectra. Thomason writes $\mathbb{H}^{\cdot}(J ; E)$ for the sequence of homotopy $\operatorname{limits}_{\{}\left\{\operatorname{holim}_{J} E_{0}(j)\right.$, $\left.\operatorname{holim}_{J} E_{1}(j), \ldots\right\}$, observes that it is a spectrum and calls it hypercohomology. This terminology is inspired by the Dold-Kan correspondence between non-negative chain complexes and simplicial abelian groups; the successive truncations of any chain complex correspond to a sequence of simplicial abelian groups, forming a spectrum, and the usual hypercohomology groups of homological algebra turn out to be the homotopy groups of the corresponding spectrum $\mathbb{H}^{\prime}$.

When $J=G$ is a group (considered as a category with one object) and $E$ is a $G$-spectrum, $\mathbb{H}^{\cdot}(G ; E)$ is the same as the homotopy fixed point spectrum $E^{h G}$, and there is a canonical map from the fixed point spectrum $E^{G}$ to $E^{h G}$. Further, if $E=\operatorname{Spt}(S)$ is the spectrum associated to a symmetric monoidal category $S$ and $S^{G}$ denotes the category of $G$-representations in $S$, Thomason's homotopy limit problem [T10] asks how close the canonical map from $E^{G}=\operatorname{Spt}\left(S^{G}\right)$ to $\operatorname{Spt}(S)^{h G}$ is to a homotopy equivalence. When $S$ is the category of complex vector spaces, resp. finite sets, the answer to the homotopy limit problem is given by a celebrated theorem of Atiyah, resp., of Carlsson (Segal Conjecture). When $G$ is the Galois group of a Galois extension $F$ of a field $E$, the homotopy limit problem asks how close $K(E)=K(F)^{G}$ and $K(F)^{h G}$ are. This is the crucial case of the QuillenLichtenbaum conjectures.

With this enlightenment, Thomason was able to mimic the exposition of [SGA4], using cosimplicial resolutions (with $J=\Delta$ ) to construct the sheaf hypercohomology spectrum $\mathbb{H}^{\cdot}(X ; F)$ on any space $X$, or even on any Grothendieck site such as the étale site of a scheme; see [T-EC, 1.33]. Here $F$ is a presheaf on $X$ with values in spectra, and there is a natural augmentation $F(X) \rightarrow \mathbb{H}^{\cdot}(X ; F)$. With this machinery in place, he introduced the following concept.

Definition. We say that $F$ has cohomological descent on $X$ if for every open $U$ in $X$ the augmentation $F(U) \rightarrow \mathbb{H}^{\cdot}(U ; F)$ is a weak equivalence. 
Functors satisfying cohomological descent have several nice features. For one thing, they satisfy the Mayer-Vietoris property: for every open $U$ and $V$ the square

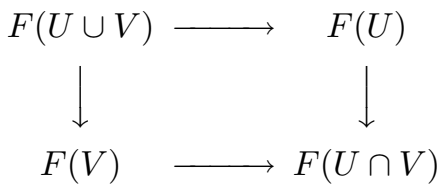

is homotopy-cartesian. When $X$ is a noetherian scheme of finite Krull dimension, cohomological descent (for the Zariski topology) is equivalent to the Mayer-Vietoris property; see [T-EC, Ex. 2.5]. A second feature is that many properties of $F(X)$ are determined by the sheaves $\tilde{\pi}_{i} F$ associated to the presheaves $U \mapsto \pi_{i} F(U)$, because there is a right half-plane "hypercohomology" spectral sequence

$$
E_{2}^{p q}=H^{p}\left(X ; \tilde{\pi}_{-q} F\right) \Rightarrow \pi_{-p-q} F(X) .
$$

This is just a disguised version of the Bousfield-Kan spectral sequence for holim [BK, §XI.7] and converges whenever $X$ has bounded cohomological dimension for the sheaves $\tilde{\pi}_{*} F$.

The most important application of all this machinery is to the $K$-theory of a scheme $X$. To avoid a discussion of the technical issues of [TT] right now, let us assume that $X$ is quasiprojective over a finite-dimensional noetherian ring. With a little care, one can construct a spectrum $K^{Q}(X)$, depending naturally upon $X$, whose homotopy groups $K_{i}(X)=\pi_{i} K^{Q}(X)$ are Quillen's $K$-theory groups [Q341] for $i \geq 0$ (and which vanish for $i<0$ ). The naturality of $K^{Q}$ means that it is a presheaf on $X$, for either the Zariski or étale topology, so we can form the Zariski and étale hypercohomology spectra $\mathbb{H}^{\cdot}\left(X ; K^{Q}\right)$ and $\mathbb{H}^{\cdot}\left(X_{\mathrm{et}} ; K^{Q}\right)$.

Suppose first that $X$ is regular. Brown and Gersten had observed as early as 1972 that the presheaf $K^{Q}$ satisfies the Mayer-Vietoris property [BG], so it has cohomological descent on $X$ for the Zariski topology. In this case, if $\tilde{K}_{q}$ denotes the sheaf associated to $K_{q}$, then $(*)$ becomes the (fourth quadrant) "Brown-Gersten" spectral sequence of $[\mathrm{BG}]$ :

$$
E_{2}^{p q}=H^{p}\left(X ; \tilde{K}_{-q}\right) \Rightarrow K_{-p-q}(X) .
$$

If $X$ is quasiprojective but not regular, there is a fringing effect in the spectral sequence (BG) for $K^{Q}$. Terms such as $H^{p}\left(X ; \tilde{K}_{0}\right)$ can be nonzero, yet $K^{Q}(X)$ has no negative homotopy groups. In effect, $K^{Q}(X)$ cannot have cohomological descent because it is the $(-1)$-connected cover of a spectrum $K(X)=K^{B}(X)$ which does have descent (by the next theorem). We shall describe $K^{B}(X)$ later on, remarking for now that if $i<0$, the groups $\pi_{i} K^{B}(X)$ are Bass' negative $K$-groups $K_{i}(X)$. If $X$ is regular, these groups vanish and $K^{B}(X)=K^{Q}(X)$.

Thomason's Zariski Descent Theorem [TT, 10.3]. Let $X$ be quasiprojective over a finite-dimensional noetherian ring. Then $K=K^{B}$ satisfies cohomological descent (for the Zariski topology). In particular, there is a convergent "BrownGersten" spectral sequence (BG) converging to the K-groups of $X$.

Until [TT], it was not known that $K^{B}(X)$ existed in this generality. However, constructions of $K^{B}(X)$ in special cases had been known for some time. For example, when $X=\operatorname{Spec}(R)$, it is the same as the non-connective Karoubi-GerstenWagoner spectra $K^{K G W}(R)$ discovered in the early 1970's; see [PW] for an elaboration. 
It is often useful to consider $K$-theory with coefficients modulo an integer $m$. These $K$-groups are written as $K_{i}(X ; \mathbb{Z} / m)$ and are the homotopy groups of a spectrum written as $(K / m)(X)$. Since we have the benefit of hindsight and [TT], we define $(K / m)(X)$ to be the smash product of $K^{B}(X)$ with the mod $m$ Moore spectrum, noting that the literature contains many variants (for $i \leq 1$ ). Such a smash product preserves cohomological descent, so Thomason's Zariski Descent Theorem implies that $(K / m)$ also satisfies cohomological descent (for the Zariski topology).

\section{The Quillen-Lichtenbaum Conjectures}

In 1974 Quillen conjectured the existence of Atiyah-Hirzebruch type spectral sequences (one for each prime $\ell$ ), relating the $K$-groups of a ring $R$ of integers in a number field $F$ to the étale cohomology groups of $X=\operatorname{Spec}\left(R\left[\frac{1}{\ell}\right]\right)$, expecting it to converge in a stable range. In this stable range, it would imply Lichtenbaum's conjectures, which relate the order of the groups $K_{i}(R)$ to the values of the zeta function $\zeta_{F}$ at negative integers, the case $i=2$ being the Birch-Tate conjecture (known up to powers of 2), asserting that $\left|K_{2}(R)\right|=\zeta_{F}(-1) w_{2}(F)$.

Quillen's conjecture was made more precise by W. Dwyer and E. Friedlander in 1981, who showed in [DF] that such Atiyah-Hirzebruch type spectral sequences converged to "étale $K$-theory" $K_{i}^{\text {et }}(X ; \mathbb{Z} / m)$, where $m=\ell^{\nu}$ and $i \in \mathbb{Z}$. This is a twisted generalized cohomology theory on the étale homotopy type of $X$, equipped with a natural comparison map $\eta_{i}: K_{i}(X ; \mathbb{Z} / m) \rightarrow K_{i}^{\text {et }}(X ; \mathbb{Z} / m)$; see $[\mathrm{F}]$. For $X=\operatorname{Spec}\left(R\left[\frac{1}{\ell}\right]\right)$, Dwyer and Friedlander showed in [DF] that $\eta_{i}$ is onto for $i \geq 2$.

Thomason observed that étale $K$-theory was periodic in $i$ and that its periodicity came from multiplication by a Bott element $\beta^{N} \in K_{2 N}(X ; \mathbb{Z} / m)$ for a suitable integer $N$; if $R$ contains a primitive $m^{\text {th }}$ root of unity, then $\beta \in K_{2}(X ; \mathbb{Z} / m)$. Thus if one inverts the Bott element, the comparison map factors through the resulting periodic groups $K_{i}(X ; \mathbb{Z} / m)\left[\beta^{-1}\right]$. Moreover, these are the homotopy groups of a spectrum $(K / m)(X)\left[\beta^{-1}\right]$. Thomason published a proof of the following theorem in [T-EC] for regular $X$ and in [TT] for general $X$.

Thomason's Étale Descent Theorem [TT, 11.5]. Suppose that $X$ is a noetherian scheme of finite Krull dimension such that all its residue fields have uniformly bounded étale cohomological dimension at the prime $\ell$. If $\ell=2$, assume that $\mathcal{O}_{X}$ contains $\sqrt{-1}$. Then the periodic theory $(K / m)\left[\beta^{-1}\right]$ satisfies étale cohomological descent for all $m=\ell^{\nu}$.

Moreover, $K_{*}(X ; \mathbb{Z} / m)\left[\beta^{-1}\right] \cong K_{*}^{\text {et }}(X ; \mathbb{Z} / m)$, via the comparison maps, and the hypercohomology spectral sequence $(*)$ is the Atiyah-Hirzebruch type spectral sequence:

$$
E_{2}^{p q}=\left\{\begin{array}{ll}
H_{\mathrm{et}}^{p}\left(X ; \mathbb{Z} / m\left(\frac{q}{2}\right)\right), & q \text { even } \\
0, & q \text { odd }
\end{array}\right\} \Rightarrow K_{-p-q}(X ; \mathbb{Z} / m)\left[\beta^{-1}\right] .
$$

Historically, the proof for $X$ regular evolved in several stages. Thomason proved étale descent in 1980, before [DF]. The surjectivity of $\eta$ (for $\ell \neq 2$ ) came in 1981 as joint work with Dwyer, Friedlander and Snaith [DFST], while the final step [T7] came in 1982, and the refinement [T9] was written in 1983. Several nice applications of the Descent Theorem were written up before the final version of [T-EC] appeared: a proof of $\mathbb{Q}_{\ell^{-}}$adic cohomological purity in [T11] and a Rigidity 
Theorem with Gillet in [GT] (their proof was contemporaneous with Gabber's). Applications to Riemann-Roch are given in [T8] and [H].

Thomason's Étale Descent Theorem solves Quillen's conjecture up to stability in $\beta$. The comparison maps $\eta_{i}: K_{i}\left(X ; \mathbb{Z} / \ell^{\nu}\right) \rightarrow K_{i}\left(X ; \mathbb{Z} / \ell^{\nu}\right)\left[\beta^{-1}\right]$ are onto for large $i$ (see [T9]) and onto for $i \geq 2$ in the arithmetic case $X=\operatorname{Spec}\left(R\left[\frac{1}{\ell}\right]\right)$ by [DF]. The guess is that $\eta_{i}$ should be an isomorphism for $i>\operatorname{cd}_{\ell}(X)$.

If $X$ is a smooth curve or surface over the complex numbers, Suslin proved in 1994 [Sus] that $\eta_{i}$ is an isomorphism for $i \geq \operatorname{dim}(X)$. Voevodsky has announced a proof (in 1996) that if $X$ is smooth over a field and $\ell=2$, then $\eta_{i}$ is an isomorphism for $i \geq \operatorname{cd}_{2} k(X)$.

\section{EQUIVARIANT ALGEBRAIC $K$-THEORY}

During 1983-1986, Thomason wrote a series of papers [T12, T13, T14, T15, T16] about the $K$-theory $G_{*}(G, X)$ of coherent $G$-modules over a $G$-scheme $X, G$ being a reductive algebraic group. (The 1991 paper [T21] also belongs to this series.) For geometric invariant theory enthusiasts, the most interesting aspect of this work was that he could establish the existence of equivariant resolutions of a coherent $G$ module $\mathcal{F}$ by $G$-vector bundles over reasonable schemes $X$ [T15]. Such resolutions had been conjectured to exist by Seshadri and others. If $G$ is a torus and $X$ is proper over a field $k$, one case of his Lefschetz-Riemann-Roch Theorem [T13] states that we have equality of Euler characteristics in the representation $\operatorname{ring} R_{k}(G)$ :

$$
\sum(-1)^{i} H^{i}(X ; \mathcal{F})=\sum(-1)^{i} H^{i}\left(X^{G} ; \tau \mathcal{F}\right)
$$

for every $\mathcal{F}$, where $X^{G}$ is the fixed-point scheme, and the $G$-module $\tau \mathcal{F}$ on $X^{G}$ is given by a standard trace formula.

If one adopts the philosophy of [T-EC] that one should go modulo $\ell^{\nu}$ and invert the Bott element, one is led to study the theory $G / \ell_{*}^{\nu}(G, X)\left[\beta^{-1}\right]$. In [T14] [T16] he showed that this horribly named theory is a sort of equivariant topological $K$ homology theory, in the sense of Atiyah and Segal — but with respect to the étale topology. In particular, if one completes this theory (à la Atiyah) with respect to the augmentation ideal of the representation ring $R_{k}(G)$, one gets the equivariant version of étale $K$-theory with supports.

\section{$K$-THEORY AND DERIVED CATEGORIES}

After Grothendieck solved the original Riemann-Roch problem for relatively projective varieties in 1958, he set about to develop a global theory of intersections of schemes and a Riemann-Roch formula for arbitrary proper morphisms $f: Y \rightarrow X$. For this one needs a direct image map $f_{*}: K_{0}(Y) \rightarrow K_{0}(X)$. If $X$ is regular, then $f_{*}$ is given by $f_{*}(E)=\sum(-1)^{i}\left[R^{i} f_{*} E\right]$. However, the individual terms in this naïve formula do not make sense for all $X$. Grothendieck observed that in spite of this, one could still make sense of the alternating sum in the derived category of complexes of vector bundles on $X$. That is, the complex $R f_{*} E$ is perfect: $X$ is covered by affine opens $\operatorname{Spec}\left(R_{j}\right)$ on which $R f_{*} E$ is represented by a bounded chain complex of finitely generated projective $R_{j}$-modules.

This being said, Grothendieck redefined $K_{0}(X)$ as a group depending upon the category $\operatorname{Perf}(X)$ of perfect complexes on $X$. However, this new definition of $K_{0}(X)$ can differ from the classical definition unless $X$ has an ample line bundle (or an "ample family" of line bundles). The book [SGA6] is devoted to making sense 
out of all this with the new definition of $K_{0}(X)$. In the end, the Riemann-Roch formula was established for proper locally complete intersection morphisms between quasi-compact schemes with an ample line bundle; see [SGA6, p. 485].

The following result of Thomason would have simplified much of [SGA6]. In this exposition, we shall assume that $X$ is noetherian, although all the following results extend to quasi-compact quasi-separated schemes, because they are inverse limits of noetherian schemes by [TT, C.9].

Key Proposition [TT, 5.2.2]. Let $U$ be open in a noetherian scheme $X$. A perfect complex $E$ on $U$ is isomorphic (in the derived category) to the restriction $E=F \mid U$ of a perfect complex $F$ on $X$ if and only if the class $[E] \in K_{0}(U)$ is in the image of the restriction map $K_{0}(X) \rightarrow K_{0}(U)$.

Moreover, if $F_{0}$ and $F_{1}$ are perfect complexes on $X$, every map $F_{0}\left|U \rightarrow F_{1}\right| U$ in the derived category is the restriction of a diagram $F_{0} \leftarrow F_{2} \rightarrow F_{1}$ of perfect complexes on $X$ with $F_{0}\left|U \cong F_{2}\right| U$.

The proof uses a result called the "Thomason-Trobaugh" direct limit characterization of perfect complexes: Let $F$ be a bounded complex on $X$ whose cohomology sheaves are quasi-coherent. Then $F$ is perfect iff for every affine open $U$ and every direct system $E_{\alpha}$ of perfect complexes on $U$, the canonical map is an isomorphism:

$$
\varliminf^{\longrightarrow} \operatorname{Hom}_{D(U)}\left(F, E_{\alpha}\right) \stackrel{\cong}{\rightrightarrows} \operatorname{Hom}_{D(U)}\left(F, \varliminf_{\longrightarrow} E_{\alpha}\right) .
$$

A word of explanation is in order. Thomason attributes the crucial idea in the proof to a dream he had on January 22, 1988, in which his recently deceased friend Thomas Trobaugh told him how to solve the final step: "The direct limit characterization of perfect complexes shows that they extend" (modulo the $K_{0}$ obstruction). In gratitude, Thomason listed his friend as a coauthor of the resulting paper $[\mathrm{TT}]$.

It is easy to extend the [SGA6] definition of $K_{0}(X)$ to higher $K$-theory. For $i \geq 0$, we define $K_{i}(X)$ to be the Waldhausen $K$-theory of the category $\operatorname{Perf}(X)$. The results of [SGA6, IV] show that $K_{i}(X)$ agrees with $K_{i}^{Q}(X)$ whenever $X$ has an ample family of line bundles. In order to extend the definition to negative $i$, Thomason used an idea of Bass: to define $K_{i-1}$, it suffices to prove that the "Fundamental Theorem" holds for $K_{i}$. Let $X[t], X\left[t^{-1}\right]$ and $X\left[t, t^{-1}\right]$ denote the products of $X$ with $\operatorname{Spec}(\mathbb{Z}[t]), \operatorname{Spec}\left(\mathbb{Z}\left[t^{-1}\right]\right)$ and $\operatorname{Spec}\left(\mathbb{Z}\left[t, t^{-1}\right]\right)$, respectively. The Fundamental Theorem we now state had been previously proven by Quillen in two important cases: for $X$ regular (in [Q341]) and for $X=\operatorname{Spec}(R)$ (in [GQ]). In turn, Quillen's results generalize an earlier result of Bass-Heller-Swan for $i=1$.

Fundamental Theorem of $K$-Theory [TT, 6.6]. If $X$ is a noetherian scheme, then for all $i$ there is an exact sequence:

$$
0 \rightarrow K_{i}(X) \rightarrow K_{i}(X[t]) \oplus K_{i}\left(X\left[t^{-1}\right]\right) \rightarrow K_{i}\left(X\left[t, t^{-1}\right]\right) \rightarrow K_{i-1}(X) \rightarrow 0 .
$$

Thomason first proved exactness for $i \geq 1$, using the Key Proposition for $X\left[t, t^{-1}\right] \subset X[t]$. Following Bass, the rest is automatic, because one inductively defines $K_{i-1}(X)$ using the Fundamental Theorem for $i$. The construction of the nonconnective spectrum $K^{B}(X)$ uses a similar induction.

We now turn to Thomason's solution of the localization problem: find the third term in the long exact sequence containing the maps $K_{i}(X) \rightarrow K_{i}(X-Z)$, where $Z$ is closed in $X$. Once again, this generalized results of Quillen: in [Q341] for $X$ 
regular and in [GQ] for $X$ affine and $Z$ a divisor. Let $K^{B}(X$ on $Z$ ) denote the (nonconnective) Waldhausen $K$-theory spectrum for the category of perfect complexes of $X$ which are acyclic off $Z$, and write its homotopy groups as $K_{i}(X$ on $Z)$.

Localization Theorem [TT, 7.4]. Let $Z$ be closed in a noetherian scheme $X$, with complement $U=X-Z$. Then the fiber of $K^{B}(X) \rightarrow K^{B}(U)$ is $K^{B}(X$ on $Z)$, and there is a long exact sequence:

$$
\cdots \rightarrow K_{i+1}(X) \rightarrow K_{i+1}(U) \rightarrow K_{i}(X \text { on } Z) \rightarrow K_{i}(X) \rightarrow K_{i}(U) \rightarrow \cdots
$$

If $X=U \cup V$, then $Z \subset V$, and the Key Proposition implies an excision result: $K(X$ on $Z) \simeq K(V$ on $Z)$; see [TT, 3.19]. By the Localization Theorem, this implies that the Mayer-Vietoris property holds for $K$-theory, which in turns proves Thomason's Zariski Descent Theorem, stated above.

Among the many applications of these structural theorems, we would like to point out the main results of [T23]. An immersion $Z \hookrightarrow X$ is called regular of codimension $d$ if $Z$ is locally defined by a regular sequence of length $d$.

Corollary [T23, 2.1]. Let $X^{\prime} \stackrel{\pi}{\rightarrow} X$ denote the blow-up of a noetherian scheme $X$ along a regular immersion $Z \stackrel{\iota}{\hookrightarrow} X$ of codimension $d$. Then

$$
K_{i}\left(X^{\prime}\right)=K_{i}(X) \oplus \bigoplus_{i=0}^{d-1} K_{i}(Z) .
$$

This result is new even for $i=0$, because the classical result of [SGA6, VII] required the extra hypothesis that $Z$ be regular. Thomason also gives an excess intersection formula for the composition $\pi^{*} \iota_{*}: K_{i}(Z) \rightarrow K_{i}\left(X^{\prime}\right)$, which for $i=0$ is [SGA6, 3.4]. Finally, he proves the following nice result.

Corollary [T23, 3.1]. Let $Z \stackrel{\iota}{\hookrightarrow} X$ be a regular immersion of noetherian schemes, with conormal bundle $N$. Then the composition $\iota^{*} \iota_{*}: K_{i}(Z) \rightarrow K_{i}(Z)$ is multiplication by the element $\lambda_{-1} N=\sum(-1)^{n}\left[\wedge^{n}(N)\right]$ of $K_{0}(Z)$.

For $i=0$ this is the "formule clef" of [SGA6, VII.2.7], while for $X$ regular it is implicit in Quillen [Q341]. Thomason further observed (unpublished) that these results allow one to prove a version of the Adams-Riemann-Roch theorem, describing the behavior of $\gamma$-filtration on $K_{i}(X)$ under a complete intersection morphism.

\section{THOMASON MODEL STRUCTURES}

In the late 1960's, Quillen had established "homotopical algebra" as a framework for doing homotopy theory in algebraic settings [Q-HA] [Q-RH]. He defined a (Quillen) model category as a category $\mathcal{C}$ equipped with three distinguished families of maps (cofibrations, fibrations and weak equivalences) satisfying five axioms (CM1-CM5). Waldhausen observed that, since these families of maps are closed under composition and contain all identity maps, it is convenient to think of them as the morphisms of three subcategories of $\mathcal{C}: \operatorname{cof}(\mathcal{C}), f i b(\mathcal{C})$ and $w e(\mathcal{C})$.

In 1995, Thomason was able to finally solve the problem, raised by Grothendieck, of lifting a homotopy structure from $\mathcal{C}$ to functor categories, such as the category of simplicial presheaves on a site. Bob's basic idea was to modify Quillen's axioms in order to obtain homological algebra as a special case. Unfortunately, he never wrote his results up. What follows is an outline of Thomason's theory, based on notes (by Barbieri-Viale and Grandis) on lectures he gave in Genova, Italy, less than one month before his death. 
Definition. A Thomason model category is a category $\mathcal{C}$ equipped with three distinguished families of maps: the "cofibrations" (written as $\longmapsto$ ), the "fibrations" (written as $\rightarrow$ ), and the "equimorphisms" (written as $\stackrel{\sim}{\rightarrow}$, and thought of as weak equivalences). These are to be closed under composition and contain all identity maps, and one writes the corresponding subcategories as $\operatorname{cof}(\mathcal{C}), f i b(\mathcal{C})$ and $e q(\mathcal{C})$ respectively. $\mathcal{C}$ must have both an initial and a terminal object, and the following self-dual axioms must be satisfied:

(TM0) Every isomorphism is in $\operatorname{cof}(\mathcal{C}), f i b(\mathcal{C})$ and $e q(\mathcal{C})$.

(TM1) The pushout of a cofibration $c$ with any map $f$ exists and is a cofibration. Moreover, if either $f$ or $c$ is an equimorphism, so is its pushout. The dual assertion for the pullback of a fibration with any map must also hold.

(TM2) (Quillen's CM2) Let $f$ and $g$ be maps such that $g f=h$ is defined. If any two of $f, g, h$ are equimorphisms, so is the third.

(TM3) (Quillen's Axiom CM3w) Any retract of an equimorphism is an equimorphism.

(TM4) (Quillen's Lifting Axiom CM4) Suppose given a commutative square

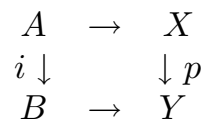

in which $i$ is a cofibration and $p$ is a fibration. Then a map $B \rightarrow X$ exists, factoring both $A \rightarrow X$ and $B \rightarrow Y$, provided either $i$ or $p$ is an equimorphism.

(TM5) (Factorization) Any map $f$ factors functorially in two ways: as $\stackrel{\sim}{\rightarrow} \rightarrow$ and as $\longmapsto \stackrel{\sim}{\rightarrow}$.

Any Quillen model category with (TM1) is a Thomason model category, provided that the factorizations required by Quillen's axiom CM5 can be made functorial; I do not know of any Quillen model category in which these factorizations are not functorial. One advantage of functoriality, already noted by Heller and Waldhausen, is that one can define "cylinder objects" $A \times I$ and "path objects" $A^{I}$ in a way that is natural in $A$.

Here is another difference between Quillen's and Thomason's axioms. Thomason's Factorization Axiom (TM5) allows us to factor $f$ as $h i$, where $i$ is a cofibration and $h$ is an equimorphism; in Quillen's Factorization Axiom (CM5) $h$ must also be a fibration. Therefore if $f$ is a map having the left lifting property with respect to all fibrations which are equimorphisms, Quillen's axioms CM3 and CM5 imply that $f$ must be a cofibration. This need not be the case in a Thomason model category, as the following example shows.

Example. Here is an example of a Thomason model category which is not a Quillen model category. Let $\mathbf{C h}$ denote the category of bounded chain complexes of modules over some ring $R$, with $e q(\mathbf{C h})$ the quasi-isomorphisms (maps inducing isomorphisms in homology).

Let $\operatorname{cof}(\mathbf{C h})$ denote the category of injections whose cokernel is a chain complex of projective modules, and let $f i b(\mathbf{C h})$ denote the category of epimorphisms whose kernel is a chain complex of injective modules. Then $\mathbf{C h}$ is a Thomason model category but not a Quillen model category (unless $R$ is a semisimple ring).

There are also two well-known Quillen model category structures on $\mathbf{C h}$ in which $e q(\mathbf{C h})$ is the class of quasi-isomorphisms; see [Q-HA, I.1.2]. In one, we retain 
$\operatorname{cof}(\mathbf{C h})$ but take the fibrations to be all epimorphisms. In the other, we retain $f i b(\mathbf{C h})$ but take the cofibrations to be all injections.

By arguments which are formally similar to Quillen's in [Q-HA], Thomason was able to reprove all the basic results of homotopical algebra for his model categories. One such basic result is that the homotopy category $\mathrm{Ho}(\mathcal{C})$ of a Thomason model category $\mathcal{C}$ exists. Recall that all equimorphisms are inverted in $\operatorname{Ho}(\mathcal{C})$ and that $\mathrm{Ho}(\mathcal{C})$ is universal with respect to this property. The existence proof follows the proof for a Quillen model category: one reduces to the problem of defining maps between a cofibrant object $A$ and a fibrant object $B$ and sets

$$
\operatorname{Hom}_{\operatorname{Ho}(\mathcal{C})}(A, B)=\operatorname{Hom}_{\mathcal{C}}(A, B) / \sim,
$$

where the homotopy relation $\sim$ is defined using the cylinder $A \times I$.

Many natural examples in Quillen's theory are enriched over simplicial sets [Q-HA, ch. II]. Quillen defines a simplicial model category to be a model category which is enriched over simplicial sets and has both "tensor" objects $X \otimes K$ and "cotensor" objects $X^{K}$ for every $X \in \mathcal{C}$ and every finite simplicial set $K$. In addition, the model structure and the simplicial structure must be compatible in the sense that the following condition must be satisfied [Q-HA, II.2.2]:

(SM7) If $A \stackrel{i}{\rightarrow} B$ is a cofibration and $p: X \longmapsto Y$ is a fibration, then

$$
\operatorname{Hom}(B, X) \rightarrow \operatorname{Hom}(A, X) \times_{\operatorname{Hom}(A, Y)} \operatorname{Hom}(B, Y)
$$

is a fibration of simplicial sets. In addition, this fibration must be an equimorphism if either $i$ or $p$ is.

Definition. A Thomason simplicial model category is a Thomason model category such that $\mathcal{C}$ is enriched over simplicial sets; has "rigid" tensor and cotensor structures which are compatible with $\operatorname{cof}(\mathcal{C}), f i b(\mathcal{C})$ and $e q(\mathcal{C})$; has good products, coproducts, limits of fibrations and colimits of cofibrations.

These additional axioms allow us to construct homotopy limits and homotopy ends internally within any Thomason simplicial model category, following the ideas of [T1]. Thomason also announced the following result, which he had been thinking about for some time in the context of Quillen model structures.

Lifting Theorem. Let $\mathcal{C}$ be a Thomason simplicial model category, I a small category. Then the functor category $\mathcal{C}^{I}$ is a Thomason simplicial model category.

Here are some remarks about the proof. If $\mathcal{C}^{I}$ is to be a Thomason simplicial model category, one must first decide what the cofibrations, etc., should be. There are at least two model structures on the functor category: a local structure and a global structure. In the global model structure, a morphism $t: F \rightarrow G$ in the functor category $\mathcal{C}^{I}$ is a cofibration or an equimorphism if each $t_{A}: F(A) \rightarrow G(A)$ is. It is a "fibration" if each $t_{A}$ is a fibration in $\mathcal{C}$ and if it has the lifting property with respect to the above notions of cofibration and equimorphism. In the local model structure, it is the cofibrations which are determined by having the lifting property with respect to the (pointwise) fibrations and equimorphisms. Of course, since these two structures have the same equimorphisms, it follows that the local and global model structures will determine the same homotopy category $\operatorname{Ho}(\mathcal{C})$.

As is usual in this subject, there is no difficulty in establishing the first few axioms, and half of axiom (TM4) is built into the definitions. The main difficulty 
in proving that this (local or global) structure forms a model category comes from the Factorization Axiom (TM5).

Here is a natural question about model structures. Suppose that we are given a (simplicial) model structure on the topos $\mathcal{C}$ of sheaves on some site $X$ and a refinement of the topology on $X$ (or any topology on $\mathcal{C}$ ). When does this induce a model structure on $\mathcal{C}_{\mathrm{sh}}$, the topos of sheaves for the refined topology? I suspect that Thomason had already studied this problem.

Thomason was also planning to deal with the stable (read triangulated) case as well, and he expressed the hope that one could lift this kind of structure to functor categories as well.

\section{REFERENCES}

[BG] K. Brown and S. Gersten, Algebraic K-theory as generalized sheaf cohomology, Lecture Notes in Math., vol. 341, Springer-Verlag, 1973, pp. 266-292, MR 50\#442.

[BK] A. Bousfield and D. Kan, Homotopy limits, completions and localizations, Lecture Notes in Math., vol. 304, Springer-Verlag, 1972, MR 51\#1825.

[BV] J. Boardman and R. Vogt, Homotopy invariant algebraic structures on topological spaces, Lecture Notes in Math., vol. 347, Springer-Verlag, 1973, MR 54\#8623a.

[DF] W. Dwyer and E. Friedlander, Étale K-theory and arithmetic, Bull. AMS 6 (1982), 453455, MR 83b:12012.

[DFST] W. Dwyer, E. Friedlander, V. Snaith and R.W. Thomason, Algebraic K-theory eventually surjects onto topological K-theory, Invent. Math. 66 (1982), 481-491, MR 84i:14015.

[F] E. Friedlander, Étale K-theory. I, Invent. Math. 60 (1980), 105-134; II, Ann. Scient. Éc. Norm. Sup. 15 (1982), 231-256, MR 82e:14029; 85c:14014.

[GQ] D. Grayson, Higher algebraic K-theory II (after D. Quillen), Lecture Notes in Math., vol. 551, Springer-Verlag, 1976, pp. 217-240, MR 58\#28137.

[GT] H. Gillet and R.W. Thomason, The K-theory of strict hensel local rings and a theorem of Suslin, J. Pure Appl. Alg. 34 (1984), 241-254, MR 86e:18014.

[H] M. Harada, A proof of the Riemann-Roch theorem, Ph.D. thesis, Johns Hopkins University, Baltimore, 1987.

[LTW] D. Latch, R.W. Thomason and S. Wilson, Simplicial sets from categories, Math. Zeit. 164 (1979), 195-214, MR 80e:55012.

[M] J.P. May, The geometry of iterated loop spaces, Lecture Notes in Math., vol. 271, SpringerVerlag, 1972, MR 54\#8623b.

[MT] J.P. May and R.W. Thomason, The uniqueness of infinite loop space machines, Topology 17 (1978), 205-224, MR 80g:55015.

[PW] E. Pedersen and C. Weibel, K-theory homology of spaces, Lecture Notes in Math., vol. 1370, Springer-Verlag, 1989, pp. 346-361, MR 90m:55007.

[Q-HA] D. Quillen, Homotopical Algebra, Lecture Notes in Math., vol. 43, Springer-Verlag, 1967, MR 36\#6480.

[Q-RH] D. Quillen, Rational homotopy theory, Annals Math. 90 (1969), 205-295, MR 41\#2678.

[Q341] D. Quillen, Higher Algebraic K-theory I, Lecture Notes in Math., vol. 341, SpringerVerlag, 1973, pp. 85-147, MR 49\#2895.

[Seg] G. Segal, Categories and cohomology theories, Topology 13 (1974), 293-312, MR 50\#5782.

[SGA4] M. Artin, A. Grothendieck and J.-L. Verdier, Théorie de Topos et Cohomologie Étale des Schémas (SGA 4), Lecture Notes in Math., vols. 269,270,305, Springer-Verlag, 1972-73.

[SGA6] P. Berthelot, A. Grothendieck and L. Illusie, Théorie des Intersections et Théorème de Riemann-Roch (SGA 6), Lecture Notes in Math., vol. 225, Springer-Verlag, 1971, MR 50\#7133.

[Sus] A. Suslin, Algebraic K-theory and Motivic Cohomology, Proc. International Congress of Mathematicians, Zürich 1994, vol. 1, Birkhäuser, 1995, pp. 342-351.

[T0] R.W. Thomason, A note on spaces with normal product with some compact space, Proc. AMS 44 (1974), 509-510, MR 51\#4165. 
[T-th] R.W. Thomason, Homotopy colimits in Cat, with applications to algebraic $K$-theory and loop space theory, Ph.D. thesis, 124 pages, Princeton University, 1977, available from University Microfilms, Ann Arbor, MI 48104.

[T1] R.W. Thomason, Homotopy colimits in the category of small categories, Math. Proc. Cambridge Philos. Soc. 85 (1979), 91-109, MR 80b:18015.

[T2] R.W. Thomason, Uniqueness of delooping machines, Duke Math. J. 46 (1979), 217-252, MR 80e:55013.

[T3] R.W. Thomason, Cat as a closed model category, Cahiers Top. Geom. Diff. 21 (1980), 305-324, MR 82b:18005.

[T4] R.W. Thomason, First quadrant spectral sequences in algebraic K-theory, Lecture Notes in Math., vol. 763, Springer-Verlag, 1979, pp. 332-355, MR 81c:18018.

[T5] R.W. Thomason, First quadrant spectral sequences in algebraic K-theory via homotopy colimits, Comm. Algebra 10 (1982), 1589-1668, 83k:18006.

[T6] R.W. Thomason, Beware the phony multiplication on Quillen's $\mathcal{A}^{-1} \mathcal{A}$, Proc. AMS 80 (1980), 569-573, MR 81k:18010.

[T-EC] R.W. Thomason, Algebraic K-theory and étale cohomology, Ann. Scient. Éc. Norm. Sup. 18, $4^{e}$ série (1985), 437-552; erratum, vol. 22, 1989, pp. 675-677, MR 87k:14016; errata $91 \mathrm{j}: 14013$.

[T7] R.W. Thomason, The Lichtenbaum-Quillen conjecture for $K / \ell_{*}\left[\beta^{-1}\right]$, Current Trends in Algebraic Topology, CMS Conf. Proc. vol. 2, Part 1, 1982, pp. 117-139, MR 84f:18024.

[T8] R.W. Thomason, Riemann-Roch for algebraic versus topological K-theory, J. Pure Appl. Alg 27 (1983), 87-109, MR 85c:14013.

[T9] R.W. Thomason, Bott stability in algebraic K-theory, AMS Contemp. Math., vol. 55, 1986, pp. 389-406, MR 87m:18022.

[T10] R.W. Thomason, The homotopy limit problem, AMS Contemp. Math., vol. 19, 1983, pp. 407-419, MR 84j:18012.

[T11] R.W. Thomason, Absolute cohomological purity, Bull. Soc. Math. France 112 (1984), 397-406, MR 87e:14018.

[T12] R.W. Thomason, Algebraic K-theory of group scheme actions, Annals of Math. Study 113 (1987), 539-563, MR 89c:18016.

[T13] R.W. Thomason, Lefschetz-Riemann-Roch theorem and coherent trace formula, Invent. Math. 85 (1986), 515-543, MR 87j:14028.

[T14] R.W. Thomason, Comparison of equivariant algebraic and topological $K$-theory, Duke Math. J. 53 (1986), 795-825, MR 88h:18011.

[T15] R.W. Thomason, Equivariant resolution, linearization and Hilbert's fourteenth problem over arbitrary base schemes, Adv. Math. 65 (1987), 16-34, MR 88g:14045.

[T16] R.W. Thomason, Equivariant algebraic vs. topological K-homology Atiyah-Segal-style, Duke Math. J. 56 (1988), 589-636, MR 89f:14015.

[T17] R.W. Thomason, The finite stable homotopy type of some topoi, J. Pure Appl. Alg. 47 (1987), 89-104, MR 88k:14010.

[T18] R.W. Thomason, A finiteness condition equivalent to the Tate conjecture over $\mathbb{F}_{q}$, AMS Contemp. Math., vol. 83, 1989, pp. 385-392, MR 90b:14029.

[T19] R.W. Thomason, Survey of algebraic vs. étale topological K-theory, AMS Contemp. Math., vol. 83, 1989, pp. 393-443, MR 90h:14029.

[T20] R.W. Thomason, The local to global principle in algebraic $K$-theory, Proc. International Congress of Mathematicians, Kyoto 1990, vol. 1, Springer-Verlag, 1991, pp. 381-394, MR 93e:19010.

[T21] R.W. Thomason, Une formule de Lefschetz en K-théorie équivariante algébrique, Duke Math. J. 68 (1992), 447-462, MR 93m:19007.

[T22] R.W. Thomason, Le principe de scindage et l'inexistence de K-théorie de Milnor globale, Topology 31 (1992), 571-588, MR 93j:19005.

[T23] R.W. Thomason, Les K-groupes d'un schéma éclaté et une formule d'intersection excédentaire, Invent. Math. 112 (1993), 195-215, MR 93k:19005.

[T24] R.W. Thomason, Les K-groupes d'un fibré projectif, Algebraic $K$-theory and algebraic topology, NATO ASI Series C, vol. 407, Kluwer, 1993, pp. 243-248.

[T25] R.W. Thomason, The classification of triangulated subcategories, preprint (1995), Compositio Math. (1996), to appear. 
[T26] R.W. Thomason, Symmetric monoidal categories model all connective spectra, Theory Appl. Categories 1 (1995), 78-118, (electronic journal http://www.tac.mta.ca/tac/).

[TT] R.W. Thomason and T. Trobaugh, Higher algebraic $K$-theory of schemes and of derived categories, The Grothendieck Festschrift III, Progress in Math., vol. 88, Birkhäuser, 1990, pp. 247-435, MR 92f:19001.

[TT1] R.W. Thomason and T. Trobaugh, Le théorème de localisation en K-théorie algébrique, C.R. Acad. Sci. (Paris) 307 (1988), 829-831, MR 89m:18015.

[TW] R.W. Thomason and S. Wilson, Hopf rings in the bar spectral sequence, Quart. J. Math Oxford 31 (1980), 507-511, MR 82f:55030.

[Wald] F. Waldhausen, Algebraic K-theory of spaces, Lecture Notes in Math., vol. 1126, SpringerVerlag, 1985, pp. 318-419, MR 86m:18011.

[W] C. Weibel, Robert W. Thomason (1952-1995), Notices of the AMS 43 (1996), 860-862.

[Y] D. Yao, Higher algebraic $K$-theory of admissible abelian categories and localization theorems, Ph.D. thesis, Johns Hopkins University, Baltimore, 1990.

Mathematics Department, Rutgers University, New Brunswick, NJ 08903

E-mail address: weibel@math.rutgers.edu 